\title{
Three dimensional (3D) printing
}

\author{
Created by: Paweł Fiedor ${ }^{1}$, Joanna Ortyl ${ }^{2}$ \\ 1, Cracow University of Technology, Faculty of Chemical Engineering and Technology, \\ Warszawska 24, 31-155 Cracow, Poland;; pawel.fiedor@doktorant.pk.edu.pl \\ 2, Cracow University of Technology, Faculty of Chemical Engineering and Technology, \\ Warszawska 24, 31-155 Cracow, Poland, Photo HiTech Ltd., Bobrzyńskiego 14, 30-348 Cracow, \\ Poland; jortyl@pk.edu.pl
}

Version received: 21 July 2020

check for updates

The following article introduces technologies that build 3 dimensional (3D) objects by adding layer-upon-layer of material, called also additive manufacturing technologies. Furthermore most important features supporting the conscious choice of 3D printing methods for applications in micro and nanomanufacturing were covered. The micromanufacturing method covers photopolymerisation based methods such as Stereolithography (SLA), Digital Light Processing (DLP), Liquid Crystal Display - DLP coupled method, Two-Photon Polymerisation (TPP) and Inkjet based methods. Functional photocurable materials, with magnetic, conductive or specific optical applications in the 3D printing processes were also reviewed.

\section{Introduction}

Three dimensional (3D) printing is currently an extremely important branch of Research and Development (R\&D) departments. This is because of its rapid prototyping, swift elimination of design errors, and improvements of the product at the prototyping stage. This approach significantly accelerates the implementation of new solutions without incurring significant production costs and eliminating in-production testing of underdeveloped models. Thanks to 3D printing techniques, making a prototype with complex geometry has become possible in a short time with unprecedented precision [1].

Progressive computerization of manufacturing processes introduces us to a new era called Industry 4.0. This level of smart production was enabled by significant breakthroughs in artificial intelligence, robotics, nanotechnology, and 3D printing observed in the twenty-first century. Thanks to the extreme customization and personalization of production technologies, the practice of Industry 4.0 is an observed phenomenon in every section of manufacturing processes. The application of artificial intelligence (AI) algorithms for the preparation and transformation of 3D models significantly quickens and improves the quality of 3D figures. Al has been successfully applied in printability checking, slicing acceleration, nozzle path planning, and, among others, cloud service platforms [2]. Evolution of industry models are presented in Figure 1.

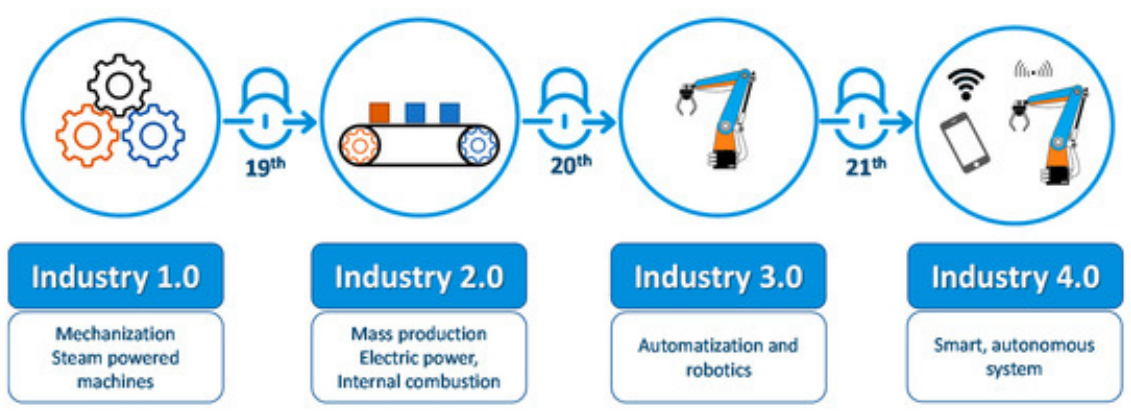

Figure 1. Industrial revolution-from Industry 1.0 to Industry 4.0. The concept of Industry 4.0 is based on encompassing a combination of traditional manufacturing and industrial platforms and practices with the latest smart technologies.

The industry has just begun to adopt additive manufacturing methods, which they use mainly to prototype and produce individual items. With Industry 4.0, 3D printing methods will be widely used to produce small batches of made-to-order products that offer construction advantages, such as complex, lightweight designs [3]. 
The flexibility of 3D printing opens the way for sharing production capacities between diverse companies for better utilization of assets and supporting growth and will also open up opportunities to share production capacities between different companies, thereby better utilizing these assets [4]. Research projects such as Horizon 2020 are focused on creating such business models in industry.

Manufacturers have implemented 3D printing technologies through seven different additive manufacturing processes: powder bed fusion, vat photopolymerization, binder jetting, material extrusion, directed energy deposition, material jetting, and sheet lamination. Each method is achieved through a different variation of 3D printing technology, which varies based on its material state, sources of light or heat, print axes, feed systems, and post-production processing [5].

- Powder bed fusion: It works by melting powder to fuse particles together. It is ideal for most types of manufacturing [6].

- Vat photopolymerization: It uses a liquid instead of a powder or filament in its build platform and is a light-activated process. It is ideal for low-run injection molds [7][].

- Directed energy deposition: It uses highly focused thermal energy delivered via a laser, electron beam, or plasma art to melt and fuse material. It is used exclusively in metal additive manufacturing [9][10].

- Material jetting: Tiny nozzles dispense droplets of a waxy photopolymer, layer by layer, which is hardened via UV light. It is ideal for items requiring high detail and high accuracy [11][12].

- Binder jetting: It is similar to material jetting but instead uses a powdered material and a binding agent. It is primarily used in furniture design models [13][14].

- Material extrusion: A thermoplastic filament is extruded through a heated nozzle onto the build platform, which solidifies as it cools. It is the most commonly referred to additive manufacturing method when someone is discussing $3 \mathrm{D}$ printing [15][16].

- Sheet lamination: Ultra-thin layers of solid material are bonded by alternating layers of adhesive. It is best used for non-functional models [17].

The history of 3D printing in its extremely turbulent beginnings revealed many ideas and solutions that have been verified by the market over time ${ }^{[18}$. Among the ideas that have been developed for commercialized technologies based on the polymerization of liquid resin are thermal processing of thermoplastic materials, melting/sintering of metals, and methods creating prints from composite materials or rubber. The list of methods that should be highlighted in the topic of 3D printing is presented below [19]:

- Photopolymerization of liquid resin:

- SLA-stereolithography

- DLP_digital light processing

- MJP-multijet printing

- BJ-binder jetting

- CAL_computed axial lithography

- SGC-solid ground curing

- CLIP_continuous liquid interface production

- Thermal processing of thermoplastic polymers:

- FDM-fused deposition modeling

- BPM-ballistic particle manufacturing

- LS-selective laser sintering

- Metal structure fabrication:

- DED—directed energy deposition

- DMLS/SLM—direct metal laser sintering

- EBM-electron-beam additive manufacturing

- MBJ-metal binder jetting

- Other methods: 
- LAM — liquid additive manufacturing deposits a liquid or highly viscous material (e.g., liquid silicone rubber) onto a build surface to create an object, which is then vulcanized using heat to harden it

- LOM-laminated object manufacturing patented by Michael Feygin—printers that cut cross-sections out of special adhesive coated paper using a carbon dioxide laser and then laminate them together

- CFF-continuous filament fabrication

\section{Photopolymerization-Based 3D Printing Methods}

In the very beginning of 3D printing, photopolymers were applied for manufacturing designed structures $\frac{20]}{\text {. To }}$ produce a three-dimensional element, flat templates with a shape corresponding to the element's cross-section were used, through which the photosetting resin was illuminated [21]. The depth of the element was obtained in the same way as in today's printers-by maneuvering the printer table up and down. The template-based solution found little widespread interest due to the limited possibilities of the formation of shapes, and the length of the process. A scheme of the machine used in this approach is presented in Figure 2. It became necessary to find a solution to obtain objects with a more complex geometry [22][23].

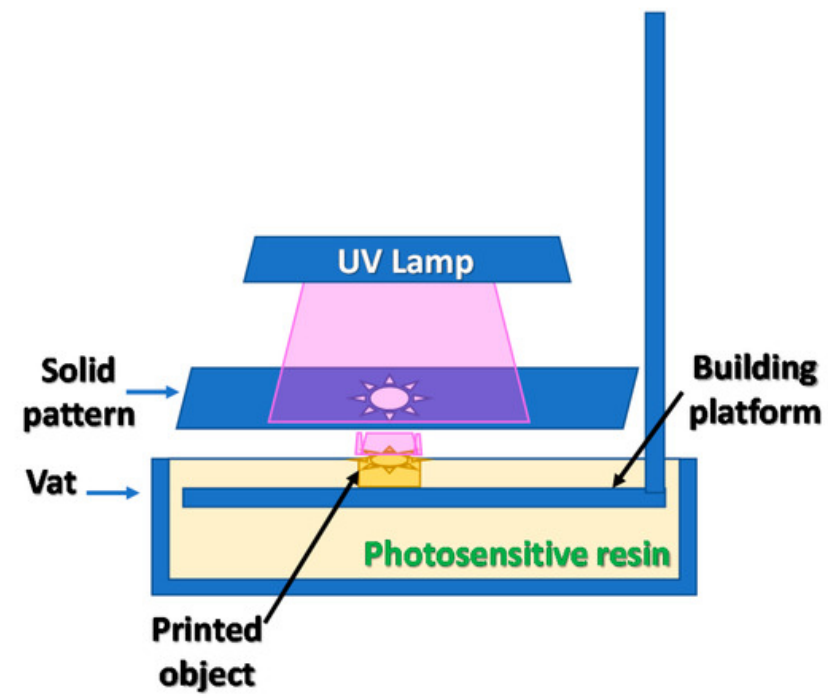

Figure 2. Origin of 3D printing; diagram of the principle of operation.

\section{Resolving power in the 3D printing processes}

The use of a laser wavelength corresponding directly to the absorption of the photoinitiator limits the maximum print resolution according to the Abbe diffraction limit. The minimum resolvable distance $\mathrm{d}$ can be calculated from the Abbe diffraction limit [24]:

$$
d=\frac{\lambda}{2 \cdot N A}
$$

Where:

NA - the numerical aperture of a laser optical system

$\lambda$ - wavelength of the laser

In the case of photopolymerisation, where a high intensity of light is required to start the process, this condition can be overcome because polymerisation starts effectively only in the centre of the laser beam, where light intensity is highest [25].

\section{Two-photon polymerisation (TPP) as a high-resolution 3D printing process}

In order to bypass the restrictions in resolving power resulting from the use of light with a wavelength of near UV and 
the lower range of visible light, it was proposed to use the two-photon absorption effect [26]. In the process of onephoton absorption, the absorbance of material is linearly dependent on the intensity of lighting, while in the case of two-photon absorption, the dependence of absorption on the square of the intensity is observed. Due to this effect, in the focus of the laser beam, only a small central area is characterised by an intensity capable of initiating two-photon polymerisation. The area with decent light intensity is therefore much smaller than when using one-photon absorption, which allows a significant improvement in resolution.

Due to the fact that the two-photon process is mediated by passing through a virtual electron state, which means that there is no energy state in the molecule corresponding to the absorption of a single photon, absorption of two photons must occur simultaneously. This means that the efficiency of the two-photon absorption process is very low. The phenomena of two-photon absorption can occur only when the sum of the energy of absorbed photons coincides with the energy difference between energetic levels of the absorbing medium. Therefore, the two-photon absorption process requires a very high intensity of lighting, and certain wavelengths with energy equal to half the energy of a single photon absorption band are favoured [26]. The basic TPP scheme is shown in figure 3.

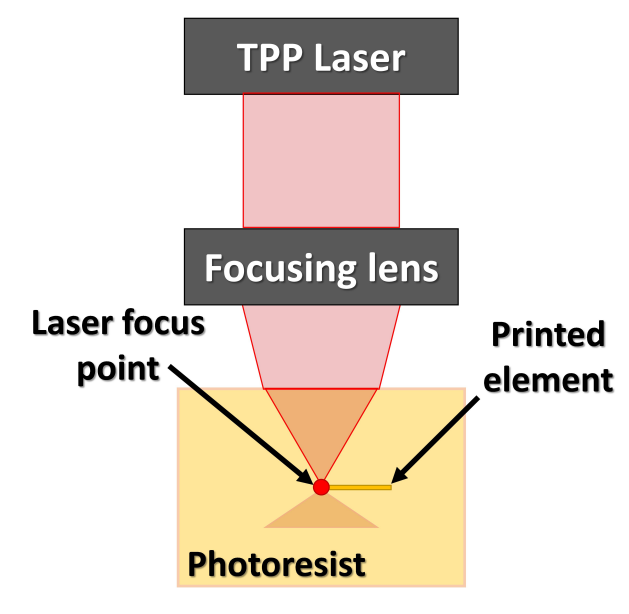

Figure 3. The two-photon polymerisation for direct writing in photoresist scheme

Using traditional broad-spectrum light sources, providing such high energy concentrated in a small area resulted in thermal destruction of the sample, which is why pulse lasers were used for TPP processes. The use of lasers with a nano or even femtosecond pulse provides the sample with an effective dose of radiation, capable of initiating the TP polymerisation process, with a small amount of total energy transferred to the sample. This allows the photopolymerisation process to take place without overheating of the sample [27]. The introduction of two-photon polymerisation into the $3 \mathrm{D}$ printing process makes it possible to obtain $3 \mathrm{D}$ objects with a size equivalent of almost $1 / 100$ of the wavelength of the light used. However, small changes in the regularity of photosensitive material and fluctuations in the intensity of the laser beam make it difficult to obtain such small objects. The development of special two-photon polymerisation initiators allows obtaining an $80 \mathrm{~nm}$ resolution using an $808 \mathrm{~nm}$ infrared laser. A reduction in the laser wavelength to $532 \mathrm{~nm}$ with a combination of the special initiating system, contributes to a further increase in resolution to about $60 \mathrm{~nm}[23]$.

\section{Conclusions}

Additive manufacturing methods are widely used in all industries requiring rapid prototyping. Thanks to the implementation of 3D scanning and computer-aided design methods, translation of the computer project into a real 3D printed model is now easier than ever before ${ }^{[28]}$. Prototype or 3D printed product development is now possible on a scale from several nanometers up to multimeter 3D prints. Depending on chosen method, fabricated objects will differ in shape accuracy and general resolution starting from methods with low resolution methods like FDM with up to 100 $\mu \mathrm{m}$ resolution, to SLA and DLP method with accuracy up to of several micrometers, and to nanoscale 3D printing with TPP techniques. On the other hand with rising accuracy, printing speed slows down to level of days needed for fabrication of tiny element with nanoscopic resolution. Thanks to recent development in two photon polymerization, printing speed can be radically shortened by fabrication of multiple objects simultaneously, but for now, this approach demand more expensive and more powerful light sources, and advanced optics. 
The methods of 3D printing allow models to be made from materials as diverse as flexible rubber-like materials to specific materials with conductive, magnetic or specialized optical properties, and the range of available materials rises annually. During the development of additive methods, materials with possible biological applications and hydrogel materials were also obtained. The major advantages and disadvantages of each described method are presented in Table 1. The choice of method used to make the appropriate model brings the consequences of an indirect selection of material resources, printing accuracy, maximum resolution, and the need for printing support structures, as well as printing time and the physical properties of the finished model.

Table 1. Comparison of described methods.

\begin{tabular}{|c|c|c|c|}
\hline Family & $\begin{array}{l}\text { Method } \\
\text { (Light } \\
\text { Source) }\end{array}$ & Advantages & Disadvantages \\
\hline \multirow{6}{*}{ Stereolithography } & $\begin{array}{l}\text { Direct SLA } \\
\text { (laser } 365 \text { to } \\
405 \mathrm{~nm})[\underline{26}]\end{array}$ & $\begin{array}{l}\text { Oldest and well-developed method with } \\
\text { multiple materials available. Fair resolution. }\end{array}$ & $\begin{array}{l}\text { Low printing rate, resolution limited by the } \\
\text { size of the laser beam and further with } \\
\text { Abby diffraction limit }\end{array}$ \\
\hline & $\begin{array}{l}\text { SLA-TPP } \\
\text { (laser 532- } \\
1064 \mathrm{~nm}) \\
{[28][29]}\end{array}$ & $\begin{array}{l}\text { Great resolution. The rising availability of } \\
\text { materials. }\end{array}$ & $\begin{array}{l}\text { Slow process-even the smallest models } \\
\text { created in days. }\end{array}$ \\
\hline & $\begin{array}{l}\text { SLA-TPP with } \\
\text { a dichromic } \\
\text { layer (laser } \\
532-1064 \mathrm{~nm}) \\
{[30]}\end{array}$ & Outstanding resolution of prints (a few $\mathrm{nm}$ ). & $\begin{array}{l}\text { Slow process-even the smallest models } \\
\text { created in days. The necessity for the } \\
\text { application of an additional photochromic } \\
\text { layer on top of the photoresist. }\end{array}$ \\
\hline & $\begin{array}{l}\text { SLA-STED } \\
\text { (laser 532- } \\
1064 \mathrm{~nm} \text { ) } \\
{[26][31]}\end{array}$ & Outstanding resolution of prints (few $\mathrm{nm}$ ). & $\begin{array}{l}\text { Slow process-even the smallest models } \\
\text { created in days. Tailoring of initiating- } \\
\text { inhibiting systems needed for all materials. }\end{array}$ \\
\hline & $\begin{array}{l}\text { SLA-TPP- } \\
\text { DMD (laser } \\
\text { 532-1064 nm) } \\
\text { [32 }\end{array}$ & $\begin{array}{l}\text { Great resolution. Possible fabrication of } \\
\text { multiple objects at the same time. }\end{array}$ & Underdeveloped method. \\
\hline & $\begin{array}{l}\text { SLA-TPP_- } \\
\text { Liquid crystal } \\
\text { devices (laser } \\
532-1064 \mathrm{~nm}) \\
{[33]}\end{array}$ & $\begin{array}{l}\text { Outstanding resolution. Possible fabrication of } \\
\text { multiple objects at the same time. Faster than } \\
\text { SLA-TPP-DMD }\end{array}$ & Underdeveloped method. \\
\hline $\begin{array}{l}\text { Fused deposition } \\
\text { modeling }\end{array}$ & FDM (-) & $\begin{array}{l}\text { Great availability of filaments, moderate } \\
\text { mechanical properties of printouts. }\end{array}$ & $\begin{array}{l}\text { Low-resolution method. Can cause } \\
\text { deformation of printouts during the } \\
\text { process. }\end{array}$ \\
\hline \multirow{2}{*}{$\begin{array}{l}\text { Digital Light } \\
\text { Processing }\end{array}$} & $\begin{array}{l}\text { DLP-DMD } \\
\text { projector (HID } \\
\text { lamp/LED } \\
\text { lamp) }[35]\end{array}$ & $\begin{array}{l}\text { High resolution for small models. High } \\
\text { precision for small models. }\end{array}$ & Bigger models decrease resolution. \\
\hline & $\begin{array}{l}\text { LCD-DLP } \\
(405-450 \mathrm{~nm} \\
\text { LED lamp) }[20]\end{array}$ & $\begin{array}{l}\text { High resolution. Resolution independent on } \\
\text { model size }\end{array}$ & $\begin{array}{l}\text { Low light intensity reaching resin vat, only } \\
\text { highly reactive resins can be used. }\end{array}$ \\
\hline \multirow{2}{*}{ Inkjet } & $\begin{array}{l}\text { Binder Jetting } \\
\text { (HID } \\
\text { lamp/LED } \\
\text { lamp) [36] } \\
{[37][38]}\end{array}$ & $\begin{array}{l}\text { Numerous materials available, relatively quick } \\
\text { printing process. }\end{array}$ & $\begin{array}{l}\text { Low resolution. Poor mechanical } \\
\text { properties of green printouts. }\end{array}$ \\
\hline & $\begin{array}{l}\text { Inkjet(HID } \\
\text { lamp/LED } \\
\text { lamp) }[36]\end{array}$ & $\begin{array}{l}\text { High resolution, multiple materials available. } \\
\text { Smooth surface finish. }\end{array}$ & $\begin{array}{l}\text { The high cost of printer and materials. } \\
\text { Only low viscous materials can be printed. }\end{array}$ \\
\hline
\end{tabular}




\begin{tabular}{llll}
\hline Family & $\begin{array}{l}\text { Method } \\
\text { (Light } \\
\text { Source) }\end{array}$ & Advantages & Disadvantages \\
\hline $\begin{array}{l}\text { Multijet (HID } \\
\text { lamp/LED } \\
\text { lamp) }[23]\end{array}$ & $\begin{array}{l}\text { High resolution, multiple materials available. } \\
\text { Possibility of printing with multiple materials at } \\
\text { the same time. Possible creation of colorful } \\
\text { printouts. Smooth surface finish. }\end{array}$ & $\begin{array}{l}\text { The high cost of printer and materials. } \\
\text { Only low viscous materials can be printed. }\end{array}$ \\
\hline
\end{tabular}

Fair resolution-hundreds of micrometers to tens of micrometers. High resolution-tens of micrometers to micrometers. Great resolution-single micrometers to hundreds of nanometers. Outstanding resolution-tens of nanometers to several nanometers.

\section{References}

1. Jimeng Yang; Yi Chen; Weidong Huang; Yun Li; Survey on artificial intelligence for additive manufacturing. 201723 rd International Conference on Automation and Computing (ICAC) 2017, 1, 1-6, 10.23919/iconac.2017.8082053.

2. Anitha Varghese; Deepaknath Tandur; Wireless requirements and challenges in Industry 4.0. 2014 International Conference on Contemporary Computing and Informatics (IC3I) 2014, 1, 634-638, 10.1109/ic3i.2014.7019732.

3. Simon Ford; Tim Minshall; Invited review article: Where and how 3D printing is used in teaching and education. Additive Manufacturing 2019, 25, 131-150, 10.1016/j.addma.2018.10.028.

4. Xiaohong Wang; Advanced Polymers for Three-Dimensional (3D) Organ Bioprinting. Micromachines 2019, 10, 814, 10.3390/mi10120814.

5. Wayne King; Holly D. Barth; Victor M. Castillo; Gilbert F. Gallegos; John Gibbs; Douglas E. Hahn; Chandrika Kamath; Alexander M. Rubenchik; Observation of keyhole-mode laser melting in laser powder-bed fusion additive manufacturing. Journal of Materials Processing Technology 2014, 214, 2915-2925, 10.1016/j.jmatprotec.2014.06.005.

6. Donald Jr Aduba; Evan D. Margaretta; Alexandra E.C. Marnot; Katherine V. Heifferon; Wyatt R. Surbey; Nicholas Chartrain; Abby R. Whittington; Timothy E. Long; Christopher B. Williams; Vat photopolymerization 3D printing of acid-cleavable PEG-methacrylate networks for biomaterial applications. Materials Today Communications 2019, 19, 204-211, 10.1016/j.mtcomm.2019.01.003.

7. Mika Salmi; Inigo Flores Ituarte; Sergei Chekurov; Eero Huotilainen; Effect of build orientation in 3D printing production for material extrusion, material jetting, binder jetting, sheet object lamination, vat photopolymerisation, and powder bed fusion. International Journal of Collaborative Enterprise 2016, 5, 218, 10.1504/IJCENT.2016.082334.

8. J.C. Heigel; P. Michaleris; E.W. Reutzel; Thermo-mechanical model development and validation of directed energy deposition additive manufacturing of Ti-6Al-4V. Additive Manufacturing 2015, 5, 9-19, 10.1016/j.addma.2014.10.003.

9. Zhuqing Wang; Todd A. Palmer; Allison M. Beese; Effect of processing parameters on microstructure and tensile properties of austenitic stainless steel 304L made by directed energy deposition additive manufacturing. Acta Materialia 2016, 110, 226-235, 10.1016/j.actamat.2016.03.019.

10. Guo Dong Goh; Yee Ling Yap; Shweta Agarwala; Wai Yee Yeong; Recent Progress in Additive Manufacturing of Fiber Reinforced Polymer Composite. Advanced Materials Technologies 2018, 4, 1-22, 10.1002/admt.201800271.

11. Lindsey Bass; Nicholas Alexander Meisel; Christopher B. Williams; Exploring variability of orientation and aging effects in material properties of multi-material jetting parts. Rapid Prototyping Journal 2016, 22, 826-834, 10.1108/rpj-11-2015-0169.

12. Yun Bai; Christopher B Williams; An exploration of binder jetting of copper. Rapid Prototyping Journal 2015, 21, 177-185, 10.1108/rpj-12-2014-0180.

13. S.M. Gaytan; M.A. Cadena; H. Karim; D. Delfin; Y. Lin; D. Espalin; E. Macdonald; R.B. Wicker; Fabrication of barium titanate by binder jetting additive manufacturing technology. Ceramics International 2015, 41, 6610-6619, 10.1016/j.ceramint.2015.01.108.

14. Sang-In Park; David W. Rosen; Seung-Kyum Choi; Chad E. Duty; Effective mechanical properties of lattice material fabricated by material extrusion additive manufacturing. Additive Manufacturing 2014, 1, 12-23, 10.1016/j.addma.2014.07.002.

15. Fang Peng; Bryan D. Vogt; Miko Cakmak; Complex flow and temperature history during melt extrusion in material extrusion additive manufacturing. Additive Manufacturing 2018, 22, 197-206, 10.1016/j.addma.2018.05.015.

16. H. Aghajani Derazkola; F. Khodabakhshi; A. Simchi; Evaluation of a polymer-steel laminated sheet composite structure produced by friction stir additive manufacturing (FSAM) technology. Polymer Testing 2020, 90, 106690, 10.1016/j.polymertesting.2020.106690.

17. Caterina Balletti; Martina Ballarin; Francesco Guerra; 3D printing: State of the art and future perspectives. Journal of Cultural Heritage 2017, 26, 172-182, 10.1016/j.culher.2017.02.010.

18. Horvath, Joan. Mastering 3D Printing; Michelle Lowman, Eds.; Apress: New york, 2014; pp. 196.

19. Lauren Safai; Juan Sebastian Cuellar; Gerwin Smit; Amir A. Zadpoor; A review of the fatigue behavior of 3D printed polymers. Additive Manufacturing 2019, 28, 87-97, 10.1016/j.addma.2019.03.023.

20. Haoyuan Quan; Ting Zhang; Hang Xu; Shen Luo; Jun Nie; Xiaoqun Zhu; Photo-curing 3D printing technique and its challenges. Bioactive Materials 2020, 5, 110-115, 10.1016/j.bioactmat.2019.12.003.

21. Hull, C.W. Apparatus for production of three-dmensonal objects by stereolithography. US4575330A 1986. 
22. Jian-Yuan Lee; Jia An; Chee Kai Chua; Fundamentals and applications of 3D printing for novel materials. Applied Materials Today 2017, 7, 120-133, 10.1016/j.apmt.2017.02.004.

23. Konstantin B. Fritzler; Victor Ya. Prinz; 3D printing methods for micro- and nanostructures. Physics-Uspekhi 2019, 62, 54-69, 10.3367/ufne.2017.11.038239.

24. S Maruo; Osamu Nakamura; S Kawata; Three-dimensional microfabrication with two-photon-absorbed photopolymerization.. Optics Letters 1997, 22, 132-134.

25. Pique, Alberto; Bertsch, Arnaud; Ranaud, Philippe; Fourkas, John T.; Andrzejewska, Ewa; . Three-dimensional microfabrication using two-photon polymerization; Baldacchini, Tommaso, Eds.; William Andrew: Elsevier, 2016; pp. 368.

26. J. Serbin; A. Egbert; Andreas Ostendorf; Boris Chichkov; R. Houbertz; G. Domann; J. Schulz; C. Cronauer; L. Fröhlich; M. Popall; et al. Femtosecond laser-induced two-photon polymerization of inorganic-organic hybrid materials for applications in photonics.. Optics Letters 2003, 28, 301-303, 10.1364/ol.28.000301.

27. Qingchuan Guo; Reza Ghadiri; Thomas Weigel; Andreas Aumann; Evgeny L. Gurevich; Cemal Esen; Olaf Medenbach; Wei Cheng; Boris Chichkov; Andreas Ostendorf; et al. Correction: Guo, Q.; et al. Comparison of in Situ and ex Situ Methods for Synthesis of Two-Photon Polymerization Polymer Nanocomposites. Polymers 2014, 6, 2037-2050. Polymers 2014, 6, 2433-2434, 10.3390/polym6092433.

28. Joanna Ortyl; Paweł Fiedor; Anna Chachaj-Brekiesz; Maciej Pilch; Emilia Hola; Mariusz Galek; The Applicability of 2-amino-4,6diphenyl-pyridine-3-carbonitrile Sensors for Monitoring Different Types of Photopolymerization Processes and Acceleration of Cationic and Free-Radical Photopolymerization Under Near UV Light.. Sensors 2019, 19, 1668, 10.3390/s19071668.

29. Minin, Igor; Minin, Oleg. Diffractive Optics and Nanophotonics Resolution Below the Diffraction Limit; Babaev, Egor; et. all;, Eds.; Springer International Publishing: New York, 2016; pp. 7-30.

30. Pique, Alberto; Bertsch, Arnaud; Ranaud, Philippe; Fourkas, John T.; Andrzejewska, Ewa. Three-dimensional microfabrication using two-photon polymerization; Baldacchini, Tommaso, Eds.; William Andrew: Elsevier, 2016; pp. 368.

31. Trisha L. Andrew; Hsin-Yu Tsai; Rajesh Menon; Confining Light to Deep Subwavelength Dimensions to Enable Optical Nanopatterning. Science 2009, 324, 917-921, 10.1126/science.1167704.

32. Patrizia Richner; Patrick Galliker; Tobias Lendenmann; Stephan J. P. Kress; David K. Kim; David J. Norris; Dimos Poulikakos; FullSpectrum Flexible Color Printing at the Diffraction Limit. ACS Photonics 2016, 3, 754-757, 10.1021/acsphotonics.6b00131.

33. Joachim Fischer; Martin Wegener; Three-dimensional optical laser lithography beyond the diffraction limit. Laser \& Photonics Reviews 2012, 7, 22-44, 10.1002/lpor.201100046.

34. Setareh Zakeri; Minnamari Vippola; Erkki Levänen; A comprehensive review of the photopolymerization of ceramic resins used in stereolithography. Additive Manufacturing 2020, 35, 101177, 10.1016/j.addma.2020.101177.

35. Mitchell, A; Lafont, U; Hołyńska, M; Semprimoschnig, C; Additive manufacturing - A review of 4D printing and future applications. Additive Manufacturing 2018, 24, 606-626, https://doi.org/10.1016/j.addma.2018.10.038.

36. J. R. Tumbleston; D. Shirvanyants; N. Ermoshkin; R. Janusziewicz; A. R. Johnson; D. Kelly; K. Chen; R. Pinschmidt; J. P. Rolland; E. T. Samulski; et al.J. M. DeSimoneA. Ermoshkin Continuous liquid interface production of 3D objects. Science 2015, 347, 13491352, 10.1126/science.aaa2397.

37. Hadi Miyanaji; Shanshan Zhang; Austin Lassell; Amirali Zandinejad; Li Yang; Process Development of Porcelain Ceramic Material with Binder Jetting Process for Dental Applications. JOM 2016, 68, 831-841, 10.1007/s11837-015-1771-3.

38. K Thrimurthulu; Pulak M Pandey; N Venkata Reddy; Optimum part deposition orientation in fused deposition modeling. International Journal of Machine Tools and Manufacture 2004, 44, 585-594, 10.1016/j.jimachtools.2003.12.004.

39. Walczak, R; Inkjet 3D printing - towards new micromachining tool for MEMS fabrication. BULLETIN OF THE POLISH ACADEMY OF SCIENCES TECHNICAL SCIENCES 2018, 66, 179-186, 10.24425/122098.

40. Casey Check; Richard Chartoff; Sean Chang; Inkjet printing of 3D nano-composites formed by photopolymerization of an acrylate monomer. Reactive and Functional Polymers 2015, 97, 116-122, 10.1016/j.reactfunctpolym.2015.09.009.

\section{Keywords}

3D printing; High-resolution; Additive manufacturing; Photopolymerization; Industry 4.0; Stereolithography; Two-photon polymerisation

(C) 2020 by the author(s). Distribute under a Creative Commans CC BY license 